\title{
Inheritance Studies and Validation of Molecular Markers Associated with Botrytis Grey Mould in Chickpea (Cicer arietinum L.)
}

Rajneesh Bhardwaj, R.K. Panwar, A.K. Gaur, S.K. Verma, Anju Arora, Sonali Gaur, Mamta Nehra

10.18805/LR-4674

\begin{abstract}
Background: The Botrytis grey mould (BGM) is a devastating foliar disease of chickpea. In order to develop resistant high yielding varieties, the genetic mechanism governing the inheritance of resistance against BGM must be decoded. The molecular markers associated with BGM resistance are also need to be validated for marker assisted pyramiding of BGM resistance in chickpea.

Methods: The present study was conducted during rabi seasons of 2014-18 at GBPUAT, Pantnagar. The experimental material consisted of six generations $\left(\mathrm{P}_{1}, \mathrm{P}_{2}, \mathrm{~F}_{1}, \mathrm{~F}_{2}, \mathrm{BC}_{1}\right.$ and $\left.\mathrm{BC}_{2}\right)$ of a cross between a BGM resistant variety $\mathrm{GL} 10006$ and susceptible variety $\mathrm{H}$ 208. The disease data was scored on nine-point (1-9) scale and were subjected to chi-square analysis. The inheritance was also studied by using 28 STMS markers at the Pulse Breeding Laboratory of GBPUAT, Pantnagar during 2017-18. The Single Marker Analysis was performed for validation of markers associated with BGM.

Result: The results indicated that resistance for BGM in chickpea is dominant over susceptibility. A major QTL i.e. TA118 along with some minor QTLs are involved in governing resistance to BGM. The marker TA118, TS72 and TA144 can be used effectively in marker assisted selection for getting desirable recombinants in chickpea breeding.
\end{abstract}

Key words: Botrytis, Chickpea, Inheritance, Markers, Validation.

\section{INTRODUCTION}

Chickpea (Cicer arietinum L.) is a popular leguminous cool season legume crop served as a high-quality diet for human consumption as its grain are rich source of protein, essential amino acids, vitamins and minerals. Globally it is second most consumed legume crop after dry bean. During the year 2017-18, in India chickpea recorded a highest ever production of $11.23 \mathrm{Mt}$ from an area of 10.56 Mha with a record productivity level of $1063 \mathrm{~kg} / \mathrm{ha}$ (Anonymous, 2018). Madhya Pradesh (4.60 Mt) followed by Maharashtra (1.78 Mt), Rajasthan (1.67 Mt), Karnataka (0.72 Mt) Andhra Pradesh (0.59 Mt), Uttar Pradesh (0.58 Mt) and Gujarat (0.37 $\mathrm{Mt})$ are the major contributing states in chickpea production. In India, the first report of BGM on chickpea was given by Shaw and Ajrekar (1915) and later by Butler and Bisby (1931). Despite so much improvement in the productivity there was still a huge gap between the yield achieved in experimental plots and farmers' fields (Pande et al. 2006). There are several causes for the low productivity of chickpea but among them susceptibility to fungal pathogen is a major factor. Botrytis grey mould (BGM), caused by Botrytis cinerea Pers. ex. Fr., is one of the most devastating fungal disease in chickpea. The chickpea plants infected with Botrytis starts producing spores, which soon spread the disease rapidly through air (MacLeod and Sweetingham, 2000). The most severe attack of this fungus is on the flowers which leads to poor or no pod setting even if seeds are formed they remains shriveled (Knights and Siddique, 2002). The study of
Department of Genetics and Plant Breeding, G.B. Pant University of Agriculture and Technology, Pantnagar-263 145, Uttarakhand, India.

Corresponding Author: Rajneesh Bhardwaj, School of Agriculture Graphic Era Hill University Dehradun-248 002, Uttarakhand, India. Email: rajneeshbhardwaj8@gmail.com

How to cite this article: Bhardwaj, R., Panwar, R.K., Gaur, A.K., Verma, S.K., Arora, A., Gaur, S. and Nehra, M. (2021). Inheritance Studies and Validation of Molecular Markers Associated with Botrytis Grey Mould in chickpea (Cicer arietinum L.). Legume Research. DOI: 10.18805/LR-4674.

Submitted: 24-05-2021 Accepted: 24-07-2021 Online: 20-08-2021

literature suggested that there are very few reports about the inheritance of resistance against BGM. The limited reports available on genetics of BGM resistance suggests that the resistance is controlled by few genes (Tewari et al. 1985; Chaturvedi et al., 1995; Anuradha et al., 2011; Nehra et al., 2020). Chickpea is one of the pulses where major progress has been achieved in the use of marker-assisted selection, numerous simple sequence repeat (SSR) and SNP resources have been developed and the chickpea genetic map density has been considerably increased (Kaur et al., 2013). The study of inheritance of resistance and molecular markers associated to BGM would be helpful for targeting resistance against BGM. Hence in the present study efforts are being made to find out the mechanism of 
inheritance of resistance against BGM by using both field and marker data and to validate the molecular markers associated with resistance towards BGM.

\section{MATERIALS AND METHODS \\ Experimental material and field trial}

The field experiments of present study were conducted during the rabi seasons of 2014-15, 2015-16 and 2016-17 at Norman E. Borlaug, Crop Research Centre of the G. B. Pant University of Agriculture and Technology, Pantnagar (Uttarakhand), India $\left(29.5^{\circ} \mathrm{N}\right.$ and $\left.79.3^{\circ} \mathrm{E}\right)$. Crosses $(\mathrm{GL}$ $10006 \times \mathrm{H} 208$ ) was attempted using hand emasculation followed by immediate pollination during rabi 2014-15 between a BGM resistant variety GL 10006 with a BGM susceptible variety $\mathrm{H} 208$. The $\mathrm{F}_{1}$ seeds were planted in rabi 2015-16 and backcrosses were attempted with both the parents and also allowed to self-pollinate to produce seeds for $\mathrm{F}_{2}$ generation. New $\mathrm{F}_{1}$ 's was also attempted in rabi201516. All the generations viz., $P_{1}, P_{2}, F_{1}, F_{2}, B C_{1}\left(F_{1} \times G L\right.$ 10006) and $\mathrm{BC}_{2}\left(\mathrm{~F}_{1} \times \mathrm{H} 208\right)$ were sown in compact family block design during rabi 2016-17 since progeny to be tested in present experiment belong to different families, as this design facilitates comparison between progeny belonging to the same family as well as different families. The row-torow distance was maintained at $30 \mathrm{~cm}$ and plant to plant at 10-15 $\mathrm{cm}$. The standard package of practices for chickpea cultivation was followed.

\section{Disease screening and scoring}

The screening for BGM was done under natural epiphytotic conditions. The plants were inoculated by spraying a spore suspension $(50,000$ spores $/ \mathrm{ml})$ of 10-days old culture of Botrytis cinerea at the onset of flowering to create disease pressure in field. The observations were recorded when susceptible cultivars showed the maximum score of BGM. Five plants from each parent and all the plants of $F_{1}$, backcross and $F_{2}$ generations were screened in field. At reproductive stage disease was identified and data was recorded according to per cent plant parts affected by BGM. Disease data was scored for per cent plants affected on nine-point (1-9) scale given in Table 1 (Kaur et al., 2013).

\section{DNA extraction and PCR}

The molecular work was carried out in the Pulse Breeding laboratory of the Department of Genetics and Plant Breeding, GBPUA and T, Pantnagar during 2017-18. In case of inheritance study by using molecular marker data, SSR based STMS (Sequence Tagged Microsatellite Site) primers were tested for their association with Botrytis grey mould resistance genes in parents, $F_{1}$ and $F_{2}$ population of cross GL10006 $\times$ H208. The STMS markers are considered as more reliable as compared to SSR primers as they have high polymorphic information content and more suitable for high throughput automation. The choice of molecular markers was based on previous reports for their linkage with botrytis resistance genes. The markers used in present study were selected from the published source of Winter et al. (1999). A total 28 STMS primers viz., TA2,TA5, TA25, TA28, TA34, TA43, TA47, TA64, TS72, TA110, TA118, TA144, TA203, TAA137, TA20, TR29, TA43, TS12, TA29, TS57, TS72, CaSTMS7, CaSTMS23, CaSTMS24, ICCM0068, ICCM0160, ICCM0178, ICCM0242a were used for inheritance study and validation. The primers that showed polymorphism among parents were used for screening in $F_{1}$ and $F_{2}$ population. In $F_{1}$ and $F_{2}$ generation plants, if the amplified band size was similar found to resistant parent than these plants were considered as resistant and if band size was similar to susceptible parent than they are considered as susceptible. For the study of inheritance and validation of markers linked with BGM resistance gene, the $\mathrm{F}_{2}$ generation consisted of 84 individual plants of cross GL1006 x H208 was used for genotyping. Phenotyping was also done in same population. The primers were synthesized from Bangaluru GeNei Ltd. Assay Buffer, Taq DNA polymerase and dNTPs were purchased from Bangaluru GeNei Ltd., Bengaluru, India. The genomic DNA was extracted for molecular characterization studies by using the Cetyl tri- methyl ammonium bromide (CTAB) method of Doyle and Doyle (1987) with very slight modifications. A single PCR reaction contains a total volume of $10.0 \mu \mathrm{l}$ and consisted of $1.0 \mu \mathrm{l}$ DNA template $(100 \mathrm{ng} / \mu \mathrm{l}), 1.5 \mu \mathrm{l}$ Taq buffer (10X) with $15 \mathrm{mM} \mathrm{MgCl}_{2}, 0.3 \mu \mathrm{lNTPs} 10 \mathrm{mM}, 0.2 \mu \mathrm{TTaq}$ polymerase $3 \mathrm{U} / \mu \mathrm{l}$ and $1.0 \mu \mathrm{l}$ primers (50 ng) and $6 \mu \mathrm{l}$ part $\mathrm{dd}_{2} \mathrm{O}$ were used for each PCR reaction. The amplification reaction was carried out in thermocycler, Peq STAR, PEQ LAB Ltd., Eppendorf Ltd., Germany with a program for an initial denaturation at $94^{\circ}$ for five minutes followed by 35 cycles of $94^{\circ}$ for one minute, annealing temperature vary with primer $\left(50-65^{\circ}\right)$ for $1 \mathrm{~min}$, extension at $72^{\circ}$ for $1 \mathrm{~min}$ and a final extension at $72^{\circ}$ for $8 \mathrm{~min}$. Horizontal gel electrophoresis unit was used for fractionating PCR products on agarose gel (3.0\%) and $\mathrm{EtBr}$ for visualization of bands under UV Alpha-imager.

Table 1: Disease rating scale for Botrytis grey mould (BGM) in chickpea as suggested by Kaur et al., (2013).

\begin{tabular}{ll}
\hline Scale & Disease response \\
\hline 1 & Highly resistant (HR) (no infection on any part of the plant). \\
$1.1-3$ & Resistant (R) (minute water-soaked lesions on 1-5\% leaves) \\
$3.1-5$ & Moderately resistant (MR) (lesions and soft rotting on $11-25 \%$ leaves and tender shoots) \\
$5.1-7$ & Moderately susceptible (MS) (rotting and fungal growth on $41-55 \%$ of the leaves and shoots) \\
$7.1-9$ & Highly susceptible (HS) (extensive rotting and fungal growth on $71-100 \%$ of the leaves, shoots and stems \\
\hline
\end{tabular}




\section{Statistical analysis}

Mean disease score was calculated by using the following formula:

$$
\text { Mean disease score }=\frac{\sum(\text { Infection score } \times \text { frequency })}{\text { Total no. of plants }}
$$

The disease screening data obtained from field and the genotypic data obtained from $F_{1}$ and $F_{2}$ population using the molecular markers were subjected to chi-square analysis as per standard statistical procedure for disease inheritance study. In case of validation of markers linked with BGM the Single Marker Analysis was performed with the help of QTL Cartographer software version 2.5.

\section{RESULTS AND DISCUSSION} Inheritance studies by using field data

Promising and stable resistance against BGM is urgently required for developing high yielding and resistant varieties of chickpea. Inheritance of BGM was studied in parents, $F_{1}, F_{2}$ and backcross generations developed by cross GL10006 $\times$ H 208. Results of field study were presented in Table 2. The mean disease score of resistant parent GL10006 was noted as 2.8 and of susceptible parent $\mathrm{H} 208$ was 8.2. It was evident from these results that the parent GL10006 showed resistant reaction while the parent H 208 showed susceptible reaction. The $F$, plants exhibited the disease score of 4.1 and hence showed resistant reaction. These results indicated the dominance of resistance over susceptibility. The disease reaction pattern in $\mathrm{F}_{2}$ generation showed the disease score from 3 to 9 with a mean of 5.19, showing segregation for resistance. The segregation of $F_{2}$ population showed a wide range of response to $B G M$. The $\mathrm{F}_{2}$ generation data was found to be fit to the ratio of 3 (resistant): 1 (susceptible) indicated that inheritance of resistance to $B G M$ is controlled by single dominant gene. In the backcross of $F_{1}$ with resistant parent $\left(B_{1}\right)$ all plants showed resistant response while in case of $\mathrm{BC}_{2}$ plants segregate in ratio of 1 resistant: 1 susceptible. The results obtained from backcrosses as well as $F_{2}$ generation showed that resistance to $B G M$ is under control of a single dominant gene. The results of present study agree with some of the earlier reported findings, indicating that resistance to BGM is dominant over susceptibility and govern by a single major gene (Tewari et al., 1985; Bhardwaj et al., 2018; Nehra et al., 2020). The dominance nature of resistance against BGM is highly desirable as it facilitates easy incorporation and selection. However, Rewal and Grewal (1989) identified two genes with dominant and recessive epistasis (13:3 ratio) whereas Chaturvedi et al. (1995) reported duplicate dominant epistasis (15:1 ratio). To further validate the field results the molecular markers was used to confirm the obtained results.

\section{Inheritance study by using molecular markers along with marker validation}

The selection of high yielding and disease-free chickpea lines is the key criterion for future breeding programs. The identification and evaluation of chickpea BGM resistant lines

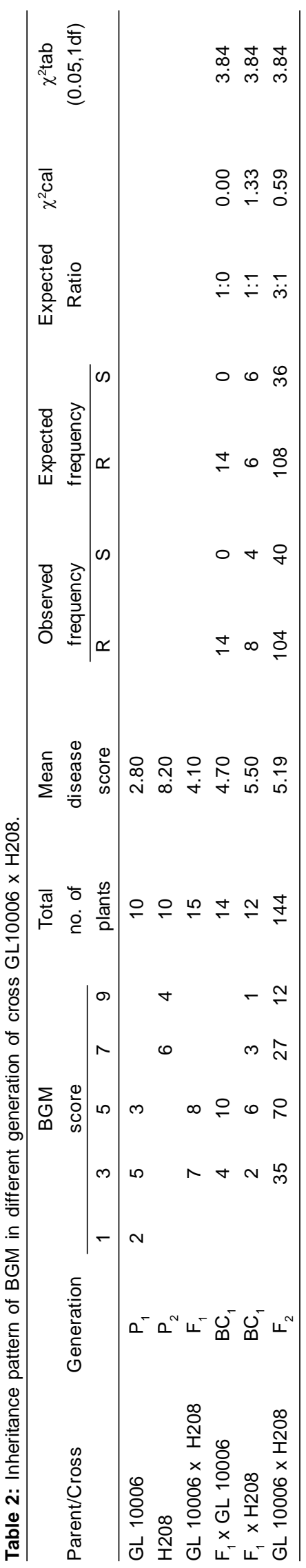

VOLUME ISSUE () 
aiming to link the marker with the specific QTL is a new avenue in chickpea breeding. In the present study, STMS primers were used to study the inheritance of resistance to BGM in $F_{1}$ and $F_{2}$ population of cross $\mathrm{GL} 10006 \times \mathrm{H} 208$ of chickpea. Out of 28 STMS primers, thirteen primers viz., TA203, TA47, TA43, TS29, TA118, TAA137, ICCM0068, TS57, ICM0160, TA34, TS72, TA25 and TA144 were found polymorphic. These polymorphic primers were screened in $F_{1}$ and $F_{2}$ population. The amplicon size for the resistant parent GL 10006 varied from $160 \mathrm{bp}$ (TA 43) to $300 \mathrm{bp}$ (ICCM 0160) whereas the amplicon size for susceptible parent $\mathrm{H} 208$ varied from $180 \mathrm{bp}$ (TA 43) to $350 \mathrm{bp}$ (TS 29) (Table 3). In $\mathrm{F}_{1}$ plants all the used primers amplified band size similar to resistant parent GL10006. These results indicated the dominance of resistance over susceptibility. In case of $F_{2}$ generation almost all the polymorphic primers showed segregation pattern that went perfectly well with the disease scoring phenotypic data for the cross GL10006 $\times \mathrm{H} 208$ (Table 4). When genotypic data was subjected to chi square analysis it showed goodness of fit in 3:1 ratio. These results were also pointing towards that the inheritance to BGM was under the control of major gene as evident from field phenotypic data. The role of major QTLs in controlling the resistance to BGM was also reported earlier by Anuradha et al. (2011) by using molecular markers in cross that involved a moderately resistant kabuli cultivar ICCV 2 and a highly susceptible desi cultivar JG 62. The thirteen polymorphic markers identified in the cross GL10006 $\times \mathrm{H}$ 208 was further subjected to single marker analysis to find the marker trait relationship. The molecular analysis was carried out in 84 plants of $F_{2}$ mapping population. The $F_{2}$ generation was also subjected to phenotyping for BGM resistance using field screening technique similarly, the genotypic data obtained was scored by using binary scoring method. The phenotypic and genotypic data obtained from $\mathrm{F}_{2}$ generation of GL10006 $\times \mathrm{H} 208$ cross was subjected to single marker analysis using Win QTL Cartographer 2.5 to detect the association of molecular marker(s) with BGM resistance locus. The results of single marker analysis of GL $10006 \times \mathrm{H} 208$ based mapping population depicted that the QTL linked with marker TA118 explained about $28.25 \%$ of total phenotypic variance whereas markers TS72, TA144, TA47, TA34, TS 57, TA 43 and TAA 137explained 7.85\%, $5.48 \%, 2.79 \%, 2.71 \%, 1.74 \%, 1.55 \%$ and $0.92 \%$ of total phenotypic variance, respectively. The marker TA118 was present on the linkage group 3 (LG 3) while the marker TS 72, TA144, TA47 and TA34, TS 57, TA 43, TAA 137 are present on linkage group 4, 3, 2, 5 and 5, respectively. The results of Single marker analysis results indicated that a major QTL i.e. TA118 along with some minor QTLs i.e. TS 72, TA 144, TA47, TA34, TS 57, TA 43, TAA 137 are involved in governing resistance to BGM. The major QTL (TA118) belongs to LG 3. The amplification profile of TA 118 and TA 144 was given in Fig 1 and 2. These results are well supported by work of Anuradha et al. (2011) which also reported three major QTLs mapped on LG 3 and LG 6 which together accounted for $43.6 \%$ of the variation for BGM resistance

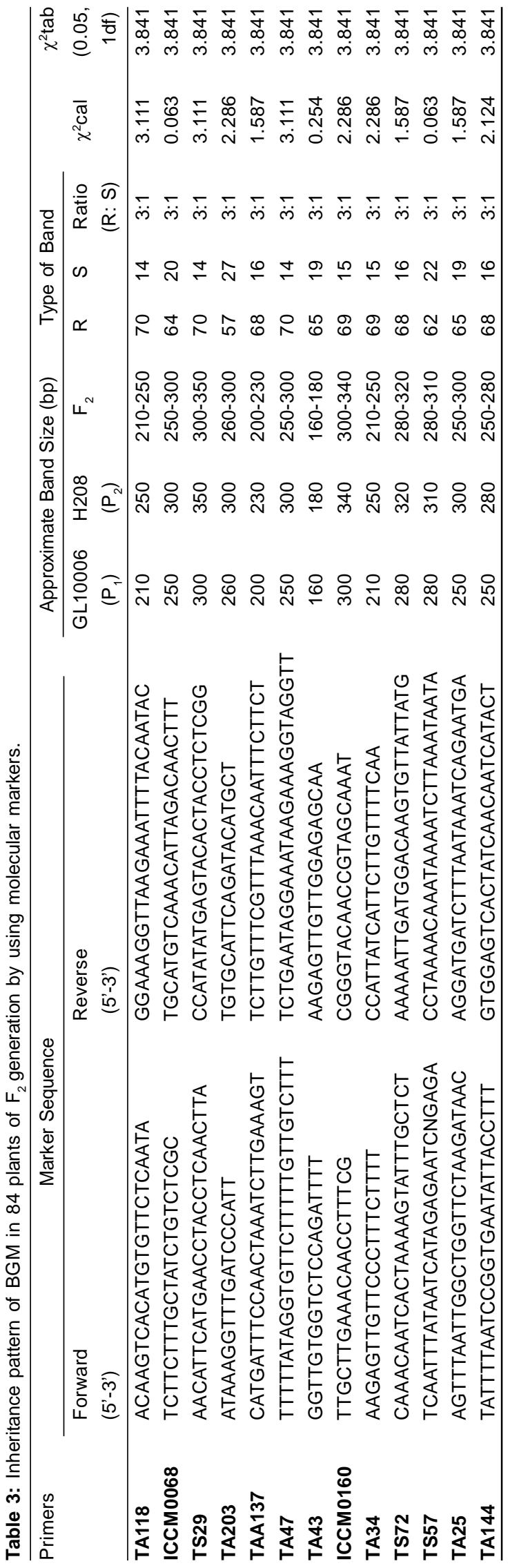

Legume Research- An International Journal 
along with some minor QTLs. On the basis of above results the marker TA118, TS72, TA144, TA47 and TA34, TS 57, TA 43, TAA 137 were found to be associated with resistance to BGM. Anuradha et al. (2011) also validated the marker TA 118 and TA144 by using recombinant inbred lines (RILs) of a cross ICCV $2 \times$ JG 62. The marker TA 144 was also validated by Ranjana et al. (2019) for Botrytis grey mould disease resistance and reported that, there is a correlation between the primers TA144 and for BGM disease resistance. Sachdeva et al. (2019) also reported that marker TA144 was strongly correlated with resistance to BGM disease. Kushwah et al. (2021) used genome wide single nucleotide polymorphism (SNPs) markers and found five QTLs viz., qbgm-3.1, qbgm-4.1, qbgm-4.2, qbgm-5.1 and qbgm-6.1 to be associated with resistance to BGM on chromosomes 3 , 4,5 and 6. Out of these five QTLs, qbgm-4.1, qbgm-4.2 and qbgm-5.1, were found to be most consistent. Their study also indicated that both major and minor QTLs were involved in governing resistance to BGM and most of these QTLs were present on linkage group 3, 4 and 5 . The above

TA 118
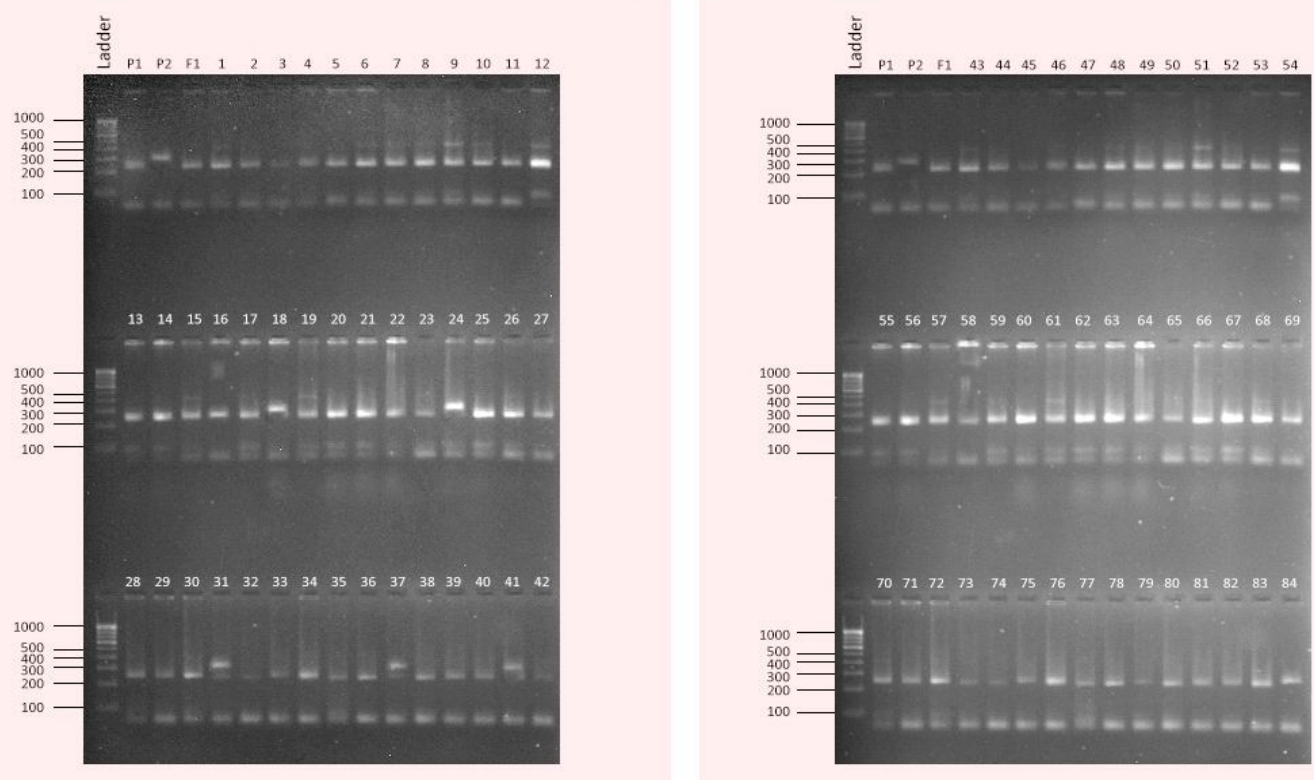

Fig 1: Amplification profile of primer TA118 in parents, $F_{1}$ and $F_{2}$ population in cross GL10006 x H208.

TA 144
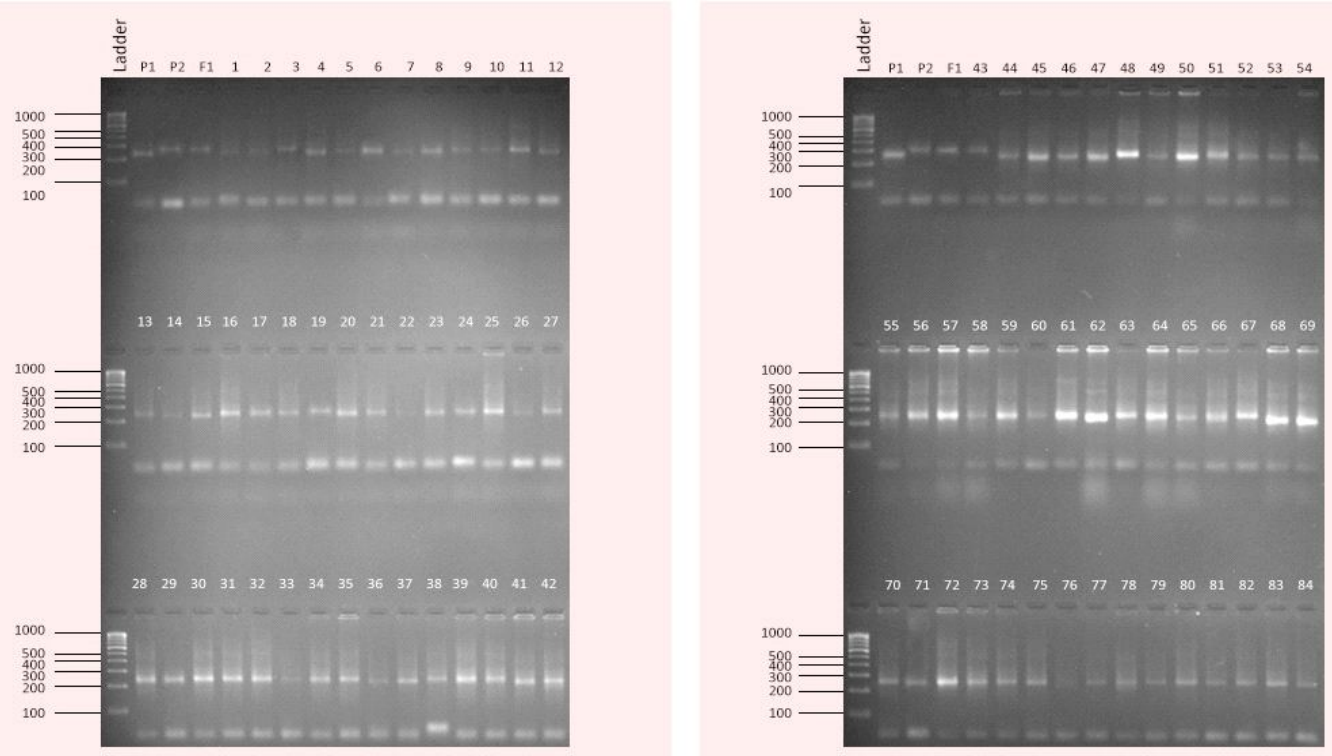

Fig 2: Amplification profile of primer TA144 in parents, $F_{1}$ and $F_{2}$ population in cross GL10006 x H208. 
Table 4: Single marker analysis results of GL10006 × H208.

\begin{tabular}{lccc}
\hline Marker & Chromosome & P-value & $\mathrm{R}^{2}$ \\
\hline TA203 & 1 & 0.66408 & 0.58 \\
TA43 & 1 & 0.46880 & 1.55 \\
TAA137 & 1 & 0.46880 & 0.92 \\
TA47 & 2 & 0.46034 & 2.79 \\
ICCM0160 & 2 & 0.46034 & 0.20 \\
TA144 & 3 & $0.00041^{* * *}$ & 5.48 \\
TA25 & 3 & $0.00041^{* * *}$ & 0.05 \\
TA118 & 3 & $0.00041^{* * *}$ & 28.25 \\
ICCM0068 & 4 & $0.02154^{*}$ & 0.39 \\
TS72 & 4 & $0.02154^{*}$ & 7.85 \\
TA29 & 5 & 0.42672 & 0.40 \\
TA34 & 5 & 0.42672 & 2.71 \\
TS57 & 5 & 0.42672 & 1.74 \\
\hline
\end{tabular}

${ }^{*},{ }^{* *},{ }^{* * *}$ significant at $5 \%, 1 \%$ and $0.01 \%$ level of significance, respectively.

discussion indicated that the validated STMS markers identified from present study will be useful in marker-assisted selection of desirable recombinants in resistance improvement programmes of chickpea.

\section{CONCLUSION}

Development of resistant varieties against BGM is must for obtaining the higher yield in chickpea. In this context it becomes very important to understand the nature of inheritance of resistance of BGM. The findings of present study indicated that the resistance against BGM is dominance over susceptibility. The results of Single marker analysis results indicated that a major QTL i.e. TA118 along with some minor QTLs i.e. TS 72, TA 144, TA47, TA34, TS 57, TA 43 and TAA 137 are involved in governing resistance to BGM. The major QTL (TA118) belongs to LG 3. On the basis of above results it can be concluded that the marker TA118, TS72 and TA144 can be used effectively in marker assisted selection for desirable recombinants in chickpea breeding.

\section{ACKNOWLEDGMENT}

The authors are highly thankful to the Joint Director CRC and Director Experiment Station, Pantnagar for providing the necessary resources for the present study.

\section{REFERENCES}

Anonymous (2018). Pulses Revolution-From food to Nutritional security. Government of India. Ministry of Agriculture and farmer welfare, Krishi Bhawan, New Delhi. 122 p.

Anuradha, C., Gaur, P.M., Pande, S., Gali, K.K., Ganesh, M., Kumar, J. and Varshney, R.K. (2011). Mapping QTL for resistance to Botrytis grey mould in chickpea. Euphytica. 182(1): 1-9.

Bhardwaj, R., Panwar, R.K., Nehra, M., Gaur, A.K., Verma, S.K. and Arora, A. (2018). Inheritance studies on resistance to botrytis grey mould in chickpea (Cicer arietinum L.). International Journal of Chemical Studies. 6(6): 2255-2257.
Butler, E.J., Bisby, G.R. (1931). The fungi of India. Sci. Monograph No. 1.' pp. XVIII237. (ICAR: New Delhi).

Chaturvedi, R., Singh, I.S. and Gupta, A.K. (1995). Inheritance of resistance to Botrytis grey mould in chickpea (Cicer arietinum). Legume Research. 18: 1-4.

Doyle, J.J. and Doyle, J.L. (1987). A rapid DNA isolation procedure for small quantities of fresh leaf tissue. Phytochemical Bulletin. 19: 11-15

Kaur, L., Sirari, A., Kumar, D., Sandhu, J.S, Singh, S. et al. (2013). Combining Ascochyta blight and Botrytis grey mould resistance in chickpea through interspecific hybridization. Phytopathologia Mediterranea. 52(1): 157-165.

Knights, E.J., Siddique, K.H.M. (2002). Manifestation of Botrytis cinerea on Chickpeas in Australia. In: Workshop Proceedings Integrated Management of Botrytis Grey Mould of Chickpea in Bangladesh and Australia, Bangladesh Agricultural Research Institute. Joydebpur, Gazipur, Bangladesh, pp 70-77.

Kushwah, A., Bhatia, D., Rani, U., Yadav, I.S., Singh, I., Bharadwaj C. and Singh S. (2021). Molecular mapping of quantitative trait loci for ascochyta blight and botrytis grey mould resistance in an inter-specific cross in chickpea (Cicer arietinum L.) using genotyping by sequencing. Breeding Science. 71: 229-239.

MacLeod, W. and Sweetingham, M. (2000). Botrytis Grey Mould of Chickpea. In: [Regan, K., White, P., Siddique, K.H.M. (eds)], Proceedings of pulse research and industry development in Western Australia, Agriculture Western Australia, Perth, Australia, pp 87-88.

Nehra, M., Panwar, R.K., Arora, A., Verma, S.K., Bhardwaj, R. and Choudhary, R. (2020). Genetics of resistance of chickpea (Cicer arietinum L.) against Botrytis grey mould Disease. Chemical Science Review and Letters. 9(35): 740-745.

Pande, S., Galloway, J., Gaur, P.M., Siddique, K.H.M., Tripathi, H.S., et al. (2006). Botrytis grey mould of chickpea: A review of biology, epidemiology and disease management. Australian Journal of Agricultural Research. 57: 1137-1150.

Ranjana, Arora, A., Panwar, R.K. and Verma, S.K. (2019). Molecular tagging of Botrytis grey mould disease in chickpea. Legume Research. 42: 710-714.

Rewal, N. and Grewal, J.S. (1989). Inheritance of resistance to Botrytis cinerea Pers. in Cicer arietinum L. Euphytica. 44: 61-63.

Sachdeva, S., Dawar, S., Rani, U., Patil, B.S., Soren, K.R., Singh, S., Sanwal, S.K., Chauhan, S.K. and Bharadwaj, C. (2019). Identification of SSR markers linked to Botrytis grey mould resistance in chickpea (Cicer arietinum). Phytopathologia Mediterranea. 58(2): 283-292.

Shaw, F.J.F. and Ajrekar, S.L. (1915). The Genus Rhizoctonia in India. Department of Agriculture India Botany Series. 7: 117.

Tewari, S.K., Pandey, M.P., Pandya, B.P., Chaube, H.S. and Tripathi, H.S. (1985). Inheritance of resistance to Botrytis gray mold in chickpea. International Chickpea Newsletter. 12: 11-12.

Winter, P., Pfaff, T., Udupa, S.M., Huttel, B., Sharma, P.C. et al. (1999). Characterization and mapping of sequence-tagged microsatellite sites in the chickpea (Cicer arietinm L.) genome. Molecular and General and Genetics. 296: 90-101. 